\title{
Potencial sobretratamiento con hormona tiroidea: estudio poblacional de una gran comunidad
}

Potential overtreatment with thyroid hormene: large community based study

Taylor P y col. JAMA Intern Med.2014; 174(1):32-39

\section{Objetivos}

Definir las tendencias temporales en los niveles de tirotrofina al inicio de la terapia con levotiroxina sódica y la probabilidad de desarrollar un nivel suprimido de tirotrofina luego del tratamiento.

\section{Diseño y Lugar}

Estudio retrospectivo de cohorte realizado con datos secundarios de la base datos de investigación en la práctica clínica del Reino Unido de Gran Bretaña.

\section{Pacientes}

De 52.298 individuos a quienes se les había prescripto levotiroxina entre 2001 y 2009, se obtuvo la siguiente información: 1) síntomas clínicos basales, 2) niveles de tirotrofina antes de iniciar el tratamiento (criterio de inclusión) y a los cinco años después de haberlo realizado.

Fueron excluidas las personas con antecedentes de hipertiroidismo, enfermedad hipofisaria o cirugía de tiroides; y aquellos que estaban tomando medicamentos para la tiroides en forma alterna o relacionada con el embarazo.

\section{Principales mediciones}

Mediana del nivel de tirotrofina al momento de la prescripción de levotiroxina, probabilidades de iniciar tratamiento con levotiroxina en la subpoblación con niveles iguales o menores a $10,0 \mathrm{mUI} / \mathrm{L}$ y probabilidad de desarrollar supresión o niveles bajos de tirotrofina luego de la terapia con levotiroxina.

\section{Resultado}

Entre 2001 y 2009, la mediana del nivel de tirotrofina en quienes iniciaron tratatamiento cayó desde 8,7 a 7,9 mUI / L. Luego de ajustar por los cambios demográficos, se constató mayor probabilidad de ser medicado con levotiroxona ante niveles basales iguales o menores a 10,0 mUI / L en 2009 que en 2001 (OR 1,30 (IC95\%, 1,19 a 1,42). Ver figura 1.
Figura 1: evolución temporal de los niveles de tirotrofina basales en $\mathrm{mUI} / \mathrm{L}$ (eje izquierdo de las ordenadas) al inicio del tratamiento con levotiroxina y de la tasa de prescripción cada 10.000 pacientes por año (eje derecho de las ordenadas).

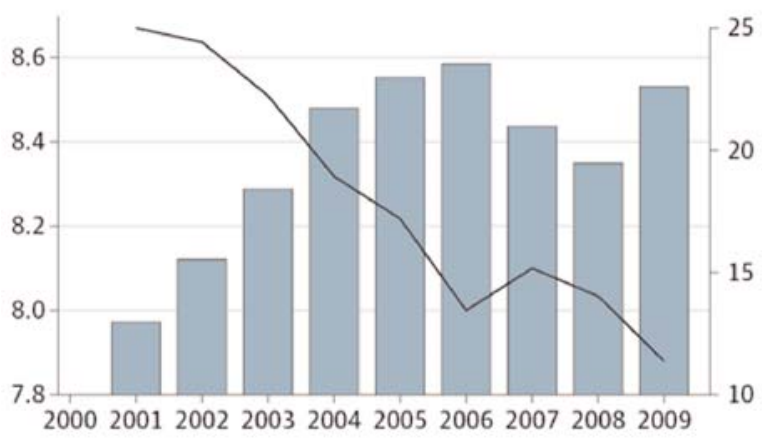

Más del $30 \%$ de los 34.808 individuos en quienes se accedió a los valores de laboratorio tenían prescripción de levotiroxina con niveles de tirotrofina iguales o menores a $10 \mathrm{mUl} / \mathrm{mL}$, valores normales de T4 y ausencia de síntomas de hipotiroidismo o factores de riesgo cardiovascular, por lo que se interpretó podían estar sobretratados.

\section{Conclusiones}

Se observó una tendencia temporal hacia el tratamiento con levotiroxina en personas con grados cada vez más marginales de hipotiroidismo y un riesgo no despreciable de desarrollar supresión de los niveles de la tirotrofina.

Fuentes de financiamiento: Consejo de Investigación Médica y Agencia Reguladora de Cuidados y Productos Médicos del Reino Unido de Gran Bretaña.

\section{Comentario}

Además de ser el hipotiroidismo primario uno de los trastornos crónicos más comunes en la población occidental, hoy existe una cultura en la cual muchos pacientes exigen que en los controles de salud se investigue su función tiroidea. Por otro lado, en la práctica habitual venimos observando cada vez con más frecuencia personas (en su mayoría mujeres) medicadas con levotiroxina. Si uno profundiza en el interrogatorio llega a la conclusión que la mayoría de estos casos "tratados" corresponden a casos de hipotiroidismo subclínico, es decir, aumento del valor de TSH con niveles normales de hormonas tiroideas y ausencia de síntomas, escenario en el cual el tratamiento con livotiroxina no coincide con las recomendaciones de los expertos ${ }^{1}$. Cabe destacar que existe cierto consen$\mathrm{so}^{2}$ en considerar la terapia con levotiroxina en individuos con niveles normales o limítrofes de tirotrofina $(10,0 \mathrm{mUl} / \mathrm{mL}$ o menos) solo en presencia de claros síntomas de hipotiroidismo, anticuerpos antitiroideos positivos, o evidencia de aterosclerosis, enfermedad cardiovascular o insuficiencia cardíaca (evidencia nivel B). Vale destacar que el sobretratamiento con levotiroxina está asocia- do con un aumento de riesgo de fracturas y fibrilación auricular, por lo que no es inocuo. Los resultados de este estudio sugieren que es generalizada la prescripción de levotiroxina en personas con niveles limítrofes de tirotrofina, práctica que podría ser perjudicial, dado el alto riesgo de desarrollar un nivel suprimido de tirotrofina después del tratamiento.

\section{Conclusión del comentador}

Mientras los especialistas en tiroides todavía están debatiendo si el hipotiroidismo subclínico debe ser tratado, cada vez es más evidente que esto ya está sucediendo a nivel poblacional. Se necesitan con urgencia ensayos clínicos controlados y aleatorizados con poder suficiente para evaluar las consecuencias en la salud del hipotiroidismo subclínico o limítrofe y de su tratamiento, con el objetivo de refinar la prescripción de levotiroxina y valorar adecuadamente el balance entre los riesgos y los beneficios de la práctica actual.

Matías Tonnelier [ IMEC (Integracion Medica de Especialidades Crespo) y Clínica Parque, Crespo, Entre Rios, mtonnelier88@gmail.com ] Tonnelier M. Potencial sobre-tratamiento con hormona tiroidea: estudio poblacional de una gran comunidad. Evid Actual Pract Ambul. 2015;18(3):81. Jul-Sep. Comentado de: Taylor P y col. Falling Threshold for Treatment of Borderline Elevated Thyrotropin Levels-Balancing Benefits and Risks: Evidence From a Large Community-Based Study. JAMA Intern Med.2014;174(1):32-39. doi:10.1001/jamainternmed.2013.11312. PMID:24100714.

\section{Referencias}

1. Royal College of Physicians. UK guidelines for the use of thyroid function tests.www.rcplondon.ac.uk/sites/default/files/the-diagnosis-and-management-of-primary-hypothyroidism-revised-statement14-june-2011_2.pdf.

2. Garber Jy col.; American Association of Clinical Endocrinologists and American Thyroid Association Taskforce on Hypothyroidism in Adults. Clinical practice guidelines for hypothyroidism in adults. Endocr Pract. 2012;18(6):988-1028. 